\title{
Lévy Process-Driven Asymmetric Heteroscedastic Option Pricing Model and Empirical Analysis
}

\author{
Gaoxun Zhang $\mathbb{D},{ }^{1}$ Yi Zheng $\mathbb{D},{ }^{2}$ Honglei Zhang $\left(\mathbb{D},{ }^{3}\right.$ and Xinchen Xie $\mathbb{D}{ }^{4}$ \\ ${ }^{1}$ School of Science, Southwest University of Science and Technology, Mianyang 621010, China \\ ${ }^{2}$ Department of Industrial Engineering, School of Construction and Management Engineering, Xihua University, \\ Chengdu 610039, China \\ ${ }^{3}$ School of Management, Yangtze Normal University, Chongqing 408100, China \\ ${ }^{4}$ College of Business, University of Illinois at Urbana-Champaign, Champaign, IL, USA
}

Correspondence should be addressed to Yi Zheng; zhengyi@mail.xhu.edu.cn

Received 4 September 2017; Accepted 21 November 2017; Published 10 January 2018

Academic Editor: Seenith Sivasundaram

Copyright (C) 2018 Gaoxun Zhang et al. This is an open access article distributed under the Creative Commons Attribution License, which permits unrestricted use, distribution, and reproduction in any medium, provided the original work is properly cited.

This paper describes the peak, fat tail, and skewness characteristics of asset price via a Lévy process. It applies asymmetric GARCH model to depict asset price's random volatility characteristics and builds a GARCH-Lévy option pricing model with random jump characteristics. It also uses circular maximum likelihood estimation technology to improve the stability of model parameter estimation. In order to test the model's pricing results, we use Hong Kong Hang Seng Index (HSI) price data and its option data to carry out empirical studies. Results prove that the pricing bias of EGARCH-Lévy model is lower than that of standard HestonNandi (HN) model in the financial industry. For short-term, middle-term, and long-term European-style options, the pricing error of EGARCH-Lévy model is the lowest.

\section{Introduction}

The Black-Scholes-Merton (BSM) option pricing model, which was constructed by Black-Scholes (1973) [1] and Merton (1973) [2], lays a fundamental theoretical framework for option pricing analysis. Lots of empirical studies have shown that "volatility smile" exists in the BSM model and it results in a big option pricing bias. Researchers attempt to build different models to reduce option pricing bias. Duan (1995) [3] used GARCH model to describe asset's random volatility and carry out option pricing analysis. Empirical studies indicate that this model can better weaken the BSW model's "volatility smile" problem. Duan (1999) [4] used the generalized error distribution (GED) to build the GARCHGED option pricing model and enlarged the GARCH option pricing model's scope of applications. Based on Duan (1999) [4], Heston and Nandi (2000) [5] built a GARCH option pricing model (HN model) that has a closed-form formula and used the HN model to carry out empirical studies for S\&P500 options. Results prove that the HN model can effectively reduce the BSM model's pricing error. Through empirical studies based on individual stock options, Stentoft (2005) [6] also discovered the HN model's advantage. By now, the HN model has become a new standard for option pricing in the financial industry [7].

However, a big bias still exists in traditional GARCH option pricing model. Therefore, it is necessary to optimize such models. Much research progress has been made in this aspect. Siu et al. (2004) [8] and Christoffersen et al. (2010) [9] built a nonnormal distribution-based GARCH option pricing model. Elliott et al. (2006) [10] introduced Markov-switching model into option pricing. Christoffersen et al. (2008) [11] built a long-term and short-term mixed volatility GARCH model. Rombouts and Stentoft (2015) [12] built a mixed normal distribution-based asymmetric GARCH model, and they found that their model was better at pricing European options through empirical studies. Chorro et al. (2015) [7] summarized recent progress of option pricing research under this framework.

In this paper, an option pricing method is created based on Esscher measure. This method allows the residual to submit to a Lévy process. Empirical studies prove that, for 
European options, this model's general error is lower than that of the HN model. In respect to short-term, middleterm, and long-term option pricing, the pricing error of Dynamic EGARCH-Lévy model is the lowest. Newey and Steigerwald (1997) [13] found that the maximum likelihood (ML) estimation method is very sensitive to the misspecifications of innovations' density. From a numerical point of view, this leads to optimization issues that grow with the number of parameters, such as local maxima. In our empirical experiments, we use another estimation approach introduced in Chorro et al. (2014) [14] which specifically aims at dealing with the potential inefficiency of the MLEs. Such efficiency issues are all the more likely to occur in our experiments as asymmetric GARCH models mixed with asymmetric conditional distributions. In addition, in order to guarantee the stability of parameter estimation, the circular maximum likelihood estimation method is used in this paper. Firstly, initial values of parameters are set according to results of existing documents to carry out maximum likelihood estimation. Then estimated results are treated as initial value of next maximum likelihood estimation. This process is done iteratively until parameters' estimation results become stable. Since the model in this paper only uses underlying assets' price data, it is suitable for Over-The-Counter (OTC) option pricing. Historical price of options contains much valuable information. In another parameter estimation method, the minimum of quadratic sum of the bias between option market price and theoretical price is used to set up an objective function. However, this method has no advantage against the GARCH option pricing model (refer to [9-12]). It is a valuable research direction to carry out GARCH option pricing analysis based on historical price information of options. Further studies will be made in the future.

Remaining parts of this paper are arranged as follows: Section 2, asymmetrical heteroscedastic Lévy process model building; Section 3, analysis of parameter estimation of asymmetrical heteroscedastic Lévy process model; Section 4, analysis of price forecast errors of options other than samples and comparison of pricing results of different option pricing models; Section 5, conclusions.

\section{Neutral Dynamic Evolution Model for Asset Risks}

$\phi_{t}$ is set as the information set at moment $t$. Assume that asset return process $R_{t}$ satisfies the following condition:

$$
R_{t}=\log \left(\frac{S_{t}}{S_{t-1}}\right)=r_{t}+m_{t}+\varepsilon_{t}
$$

where $r_{t}$ stands for risk-free interest rate; $\varepsilon_{t}$ satisfies certain conditional distribution process, that is, $\varepsilon_{t} \mid \phi_{t} \sim D\left(0, h_{t}\right)$, where $h_{t}$ stands for conditional variance satisfying $h_{t}=$ $F\left(h_{t-1}, \varepsilon_{t-1}\right)$.

Formula (1) is used in many option pricing research documents to describe asset return process but the form of $m_{t}$ varies greatly. For instance, in Duan (1995) [3], $m_{t}=\lambda_{0} \sqrt{h_{t}}-$ $h_{t} / 2$ and volatility $h_{t}$ is described via asymmetrical GARCH process. In Duan (1999) [4], $m_{t}=\lambda_{0} h_{t}$ and volatility $h_{t}$ is described via asymmetrical GARCH process. In Chorro et al. (2015) [7], $m_{t}=\lambda_{0} \sqrt{h_{t}}-h_{t} / 2$ and volatility $h_{t}$ is described via GARCH process with a leverage effect.

In Section 2.2 of this paper, Esscher martingale measure conversion technology is used to export specific form of $m_{t}$ under the condition of equivalent martingale measure and such form is used for option pricing analysis.

2.1. Lévy Process-Driven Asymmetrical Heteroscedastic Model. In this part, a dynamic asset distribution evolution process based on Lévy process and asymmetrical heteroscedastic process is built. We assume that the residual $\varepsilon_{t}$ in (1) submits to infinite-jump Lévy process and it is recorded as $\varepsilon_{t} \mid \phi_{t} \sim$ $\operatorname{Levy}\left(0, h_{t}\right)$. Because Lévy process can flexibly depict asset distribution characteristics, it has been widely applied to the description of financial asset distribution (refer to [1518]). Infinite-jump Lévy process usually has three characteristic items, $\mu, \sigma$, and $v$, representing linear drift, Brownian motion diffusion, and jump, respectively. According to LévyKhintchine formula, the relationship between the characteristic function submitted to Lévy distribution random variable $X$ and $\gamma, \sigma$, and $v$ is described as follows:

$$
\begin{gathered}
\varphi_{X}(u)=E(\exp (i u X))=\exp \left(i u \gamma-\frac{\sigma^{2} u^{2}}{2}\right. \\
\left.+\int_{-\infty}^{+\infty}\left(\exp (i u x)-1-i u x_{|x| \leq 1}\right) v d x\right) .
\end{gathered}
$$

In order to accurately depict the higher moment characteristics of asset returns, two widely applied infinite-jump Lévy processes, that is, NIG process and VG process, are taken into consideration in this paper. These two processes are reviewed as follows.

As a widely applied infinite-jump Lévy distribution, NIG distribution is put forward from [19] and it is recorded as $\operatorname{NIG}(a, \beta, \delta, \mu)$. Its characteristic function is as follows:

$$
\begin{aligned}
& \varphi_{\mathrm{NIG}}(u) \\
& \quad=\exp \left(i u \mu+\delta \sqrt{a^{2}-\beta^{2}}-\delta \sqrt{a^{2}-(\beta+i u)^{2}}\right),
\end{aligned}
$$

where $a>0,|\beta|<a, \delta>0, \mu$ and $a$ mainly control mean value and kurtosis while $\beta$ and $\delta$ mainly control skewness and secondary moment. NIG distribution's jumping term and density function may be expressed as follows:

$$
\begin{gathered}
v(d x)=\frac{\delta a}{\pi} \frac{\exp (\beta x) K_{1}(a|x|)}{|x|} d x, \\
f_{\mathrm{NIG}}(x)=\frac{\delta a}{\pi} \exp \left(\delta \sqrt{a^{2}+\beta^{2}}\right) \\
\cdot \frac{K_{1}\left(a \sqrt{\delta^{2}+(x-\mu)^{2}}\right)}{\sqrt{\delta^{2}+(x-\mu)^{2}}} \exp (\beta(x-\mu)),
\end{gathered}
$$

where $K_{1}(\cdot)$ stands for postadjustment Bessel function of the second kind. 
VG process is put forward from [15] and it is recorded as $\operatorname{VG}(\lambda, a, \beta, \mu)$ for short. Its characteristic function is as follows:

$$
\varphi_{\mathrm{VG}}(u)=\exp (i u \mu)\left(\frac{a^{2}-\beta^{2}}{a^{2}-(\beta+i u)^{2}}\right)^{\lambda},
$$

where $\lambda>0, a>|\beta| \geq 0$ and jumping term and density function may be expressed as follows:

$$
\begin{aligned}
& v(d x)=\frac{\lambda}{x}\left[\exp ((\beta-a) x) 1_{x>0}\right. \\
& \left.-\exp ((a+\beta) x) 1_{x<0}\right] d x, \\
& f_{\mathrm{VG}}(x)=\frac{\left(a^{2}-\beta^{2}\right)^{\lambda}}{\sqrt{\pi} \Gamma(\lambda)(2 a)^{\lambda-1 / 2}}|x-\mu|^{\lambda-1 / 2} \\
& \cdot K_{\lambda-1 / 2}(a|x-\mu|) \exp (\beta(x-\mu)),
\end{aligned}
$$

where $\Gamma(\cdot)$ is Gamma function.

If the conditional variance $h_{t}$ of $\varepsilon_{t}$ in formula (1) satisfies the following condition,

$$
h_{t}=\exp \left(\omega+a_{1} h_{t-1}^{-1 / 2}\left(\left|\varepsilon_{t-1}\right|-\gamma \varepsilon_{t-1}\right)\right) h_{t-1}^{b_{1}}
$$

that is, $\operatorname{EGARCH}(1,1)$ process in [18], the Lévy process satisfying formula (7) is recorded as EGARCH-Lévy process. Because EGARCH process can effectively capture asymmetrical heteroscedastic characteristics of returns, it has been widely applied to volatility modeling. Conditional heteroscedastic process (7) may also be expanded to more situations, for example, NGARCH model in [20], LMSV model in [21], and long- and short-term volatility model. Residual $\varepsilon_{t}$ distribution may be expanded to more Lévy processes, that is, CTS process in [10] and RDTS process in [18].

2.2. Neutral Dynamics of Risks and Options Worth Estimation. Usually a market is incomplete, without a unique equivalent martingale measure (EMM). In this paper, Radon-Nikodym (R-N) derivative is used to realize conversion of risk-neutral measures. In formula (8), Esscher martingale measure conversion technology is used to create R-N derivative for measure conversion:

$$
\frac{d Q_{t}}{d P_{t}} \mid \phi_{t}=\frac{e^{\theta_{t} x_{t}}}{E_{t}^{P}\left(e^{\theta_{t} x_{t}}\right)},
$$

where $d Q_{t}$ and $d P_{t}$ stand for the probability density function of random process $x_{t}$ under the risk-neutral measure and real measure, respectively; $E_{t}^{P}$ stands for the expectation under the real measure; $\theta_{t}$ stands for the conversion parameter between real measure and risk-neutral measure. In this paper, $\mathrm{R}-\mathrm{N}$ derivative is used to create underlying asset's equivalent martingale process.

According to (8), we figure out that the sufficient condition for asset prices satisfies martingale process under riskneutral measure $Q$, that is, $m_{t}$ satisfying

$$
m_{t}=\varphi_{\varepsilon_{t}}\left(\theta_{t}\right)-\varphi_{\varepsilon_{t}}\left(\theta_{t}+1\right),
$$

where $\varphi_{\varepsilon_{t}}$ stands for the logarithmic moment generating function of residual $\varepsilon_{t}$ under actual measure $P$, that is, $\varphi_{\varepsilon_{t}}(u)=\log \left(E_{t}\left(e^{u \varepsilon_{t}}\right)\right)$. In fact, if asset price satisfies the martingale process under measure $Q, S_{t-1}=e^{-r_{t}} E^{Q}\left(S_{t}\right)$, according to formulas (1) and (8), it is found that

$$
\begin{aligned}
e^{r_{t}} & =E^{Q}\left(\frac{S_{t}}{S_{t-1}}\right)=\int_{-\infty}^{+\infty} e^{R_{t}} d Q_{t} \quad \text { (Pursuant to (1)) } \\
& =\int_{-\infty}^{+\infty} e^{R_{t}} \frac{e^{\theta_{t} R_{t}}}{E^{P}\left(e^{\theta_{t} R_{t}}\right)} d P_{t} \quad \text { (Pursuant to (8)) } \\
& =\frac{\int_{-\infty}^{+\infty} e^{\left(\theta_{t}+1\right)\left(r_{t}+m_{t}+\varepsilon_{t}\right)} d P_{t}}{E^{P}\left(e^{\theta_{t}\left(r_{t}+m_{t}+\varepsilon_{t}\right)}\right)} \quad \text { (Pursuant to (1)) } \\
& =e^{r_{t}+m_{t}} \frac{E^{P}\left(e^{\left(\theta_{t}+1\right) \varepsilon_{t}}\right)}{E^{P}\left(e^{\theta_{t} \varepsilon_{t}}\right)} .
\end{aligned}
$$

After taking the logarithm and transposing terms, the following result is worked out:

$$
\begin{aligned}
m_{t} & =\log \left(E^{P}\left(e^{\theta_{t} \varepsilon_{t}}\right)\right)-\log \left(E^{P}\left(e^{\left(\theta_{t}+1\right) \varepsilon_{t}}\right)\right) \\
& =\varphi_{\varepsilon_{t}}\left(\theta_{t}\right)-\varphi_{\varepsilon_{t}}\left(\theta_{t}+1\right) .
\end{aligned}
$$

That is to say, formula (9) is tenable.

After formula (9) is put into formula (1), the evolution process of asset log returns under measure $Q$ is figured out as follows:

$$
R_{t}=r_{t}+\varphi_{\varepsilon_{t}}\left(\theta_{t}\right)-\varphi_{\varepsilon_{t}}\left(\theta_{t}+1\right)+\varepsilon_{t},
$$

where $\varepsilon_{t} \mid \phi_{t} \sim \operatorname{Levy}\left(0, h_{t}\right)$. According to formula (12), we can figure out the evolution process of asset returns under measure $Q$ as long as we get residual $\varepsilon_{t}$ which submits to $\operatorname{Levy}\left(0, h_{t}\right)$ process under measure $P$. This lays a foundation for realizing option pricing through the Monte Carlo technique.

According to the risk-neutral pricing theory, options worth is figured out as follows:

$$
C_{t}=\exp (-r(T-t)) E_{t}^{\mathrm{Q}}\left[\max \left(S_{T}-K, 0\right)\right],
$$

where $r$ stands for risk-free interest rate; $E_{t}^{Q}$ stands for random variable's expectation under measure $Q ; S_{T}$ stands for underlying asset's price under measure $Q$ at the time of $T$.

According to formulas (12) and (13), the following option value estimation formula may be worked out as follows:

$$
\begin{aligned}
C_{t}= & \exp (-r(T-t)) \frac{1}{M} \\
& \cdot \sum_{j=1}^{M} \max \left(S_{t} \exp \left(\sum_{i=t+1}^{T} R_{i}^{j}\right)-K, 0\right),
\end{aligned}
$$

where $M$ stands for the simulated path number in the Monte Carlo technique (it is assumed that $M=10000$ in this paper); $R_{i}^{j}$ stands for log returns of underlying asset at the $j$ th path under measure $Q$ and it can be figured out by sampling pursuant to formula (12). 


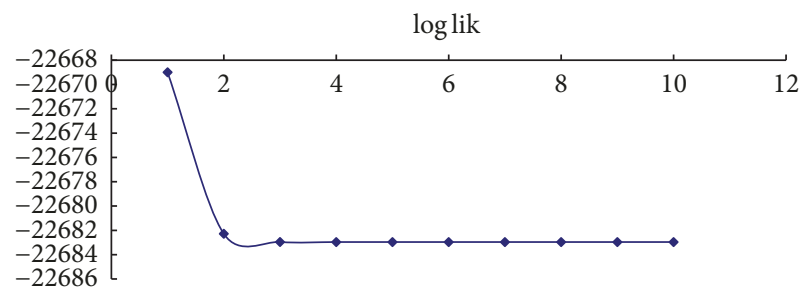

(a)

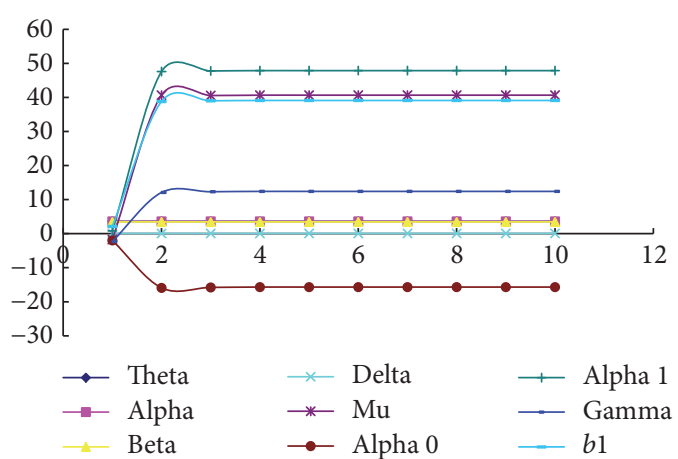

(b)

FIGURE 1: The relationship between likelihood function value of Dynamic EGARCH-Lévy-NIG model and cycle index (a) and the relationship between the parameter value of Dynamic EGARCH-Lévy-NIG model and cycle index (b).

\section{Volatility Model Parameter Estimation}

In this part, we use the daily closing price of HSI to carry out empirical studies for the EGARCH- Lévy model and the HN model. Data come from Wind database. Samples are 7,325 daily closing prices of HIS collected during the period from December 31, 1986, to July 14, 2016.

Formula (12) is used to figure out underlying asset's evolution process under measure $Q$. Formula (12) is divided into two situations according to $\theta_{t}$ value: (I) when $\theta_{t} \equiv \theta$, it is recorded as EGARCH-Lévy model; (II) when $\theta_{t}=\theta \sqrt{h_{t}}$, it is recorded as Dynamic EGARCH-Lévy model. Models in this paper may be classified as EGARCH-Lévy-NIG model, EGARCH-Lévy-VG model, Dynamic EGARCH-Lévy-NIG model, and EGARCH-Lévy-VG model.

We use Software R (R3.1.2) to complete parameter estimation. In order to reduce the estimation bias caused due to improper setting of initial values of parameters, the circular maximum likelihood estimation method is used in this paper. The first estimation result is treated as initial value of the second estimation to utilize optimization function iteratively. Iteration does not stop until likelihood function values become stable.

Figure 1 shows the relationship between the model's likelihood function value and cycle index. We only report Dynamic EGARCH-Lévy model's results. Other models' results are similar. Figure 1 indicates that, with the increase of cycle index, likelihood function value and parameter value of Dynamic EGARCH-Lévy model tend to become stable gradually and three cycles lead to optimal results.

Table 1 shows parameter estimation results of five models, that is, HN, EGARCH-Lévy-NIG, EGARCH-Lévy-NIG, EGARCH-Lévy-VG, Dynamic EGARCH-Lévy-NIG, and Dynamic EGARCH-Lévy-VG models. It indicates that, according to estimated likelihood value and BIC value, HN model performs worst and Dynamic EGARCH-LévyNIG model performs best, and EGARCH-Lévy-NIG model is better than EGARCH-Lévy-VG model. Compared with HN model, Lévy process-based asymmetrical heteroscedastic volatility model's maximum likelihood value and BIC value increase significantly, indicating that EGARCH-Lévy model can better describe dynamic process of volatility and laying a foundation for further improving the accuracy of option pricing. In addition, Table 1 shows a sequence of " $\mathrm{HN}$ model to EGARCH-Lévy-NIG model to EGARCH-Lévy-VG model" by ascending order of $\theta$ value, indicating that riskneutral measure conversion parameter values of different models vary greatly and parameter $\theta$ has a significant impact on volatility estimation.

\section{HSI Option Forecast Analysis}

In this part, empirical test will be carried out to verify forecast results of different option models. Known as a new standard in the financial industry, HN model is used as reference model to test the forecast results of four option pricing models created in this paper.

4.1. Description of Option Data. HSI option data come from the HKEX website (http://sc.hkex.com.hk/TuniS/www.hkex .com.hk/chi/index_c.htm). Samples occurred during the period from July 10, 2016, to July 31, 2017. There are up to 81 types of one-day closing prices. Options include shortterm ones (expiring within 30 days), middle-term ones (expiring in 30-60 days), and long-term ones (expiring 60 days later). Options with a short term (less than 60 days) are traded briskly. Long-term options have a limited closing price selection space. Taking into consideration actual transaction details of HSI options, sample options are divided into three types, that is, short-term options $(T<30)$, middle-term options $(30 \leq T<60)$, and long-term options $(60 \leq T)$. Inactively traded options are eliminated. Taking into consideration the weekend effect of stock prices, option data on every Wednesday are used as samples. In this way, the weekend effect is removed.

4.2. Option Price Forecast Analysis. In the process of option pricing, we use the Monte Carlo estimation technique, including four steps:

(I) Use the maximum likelihood estimation method to estimate option pricing model's parameters. 


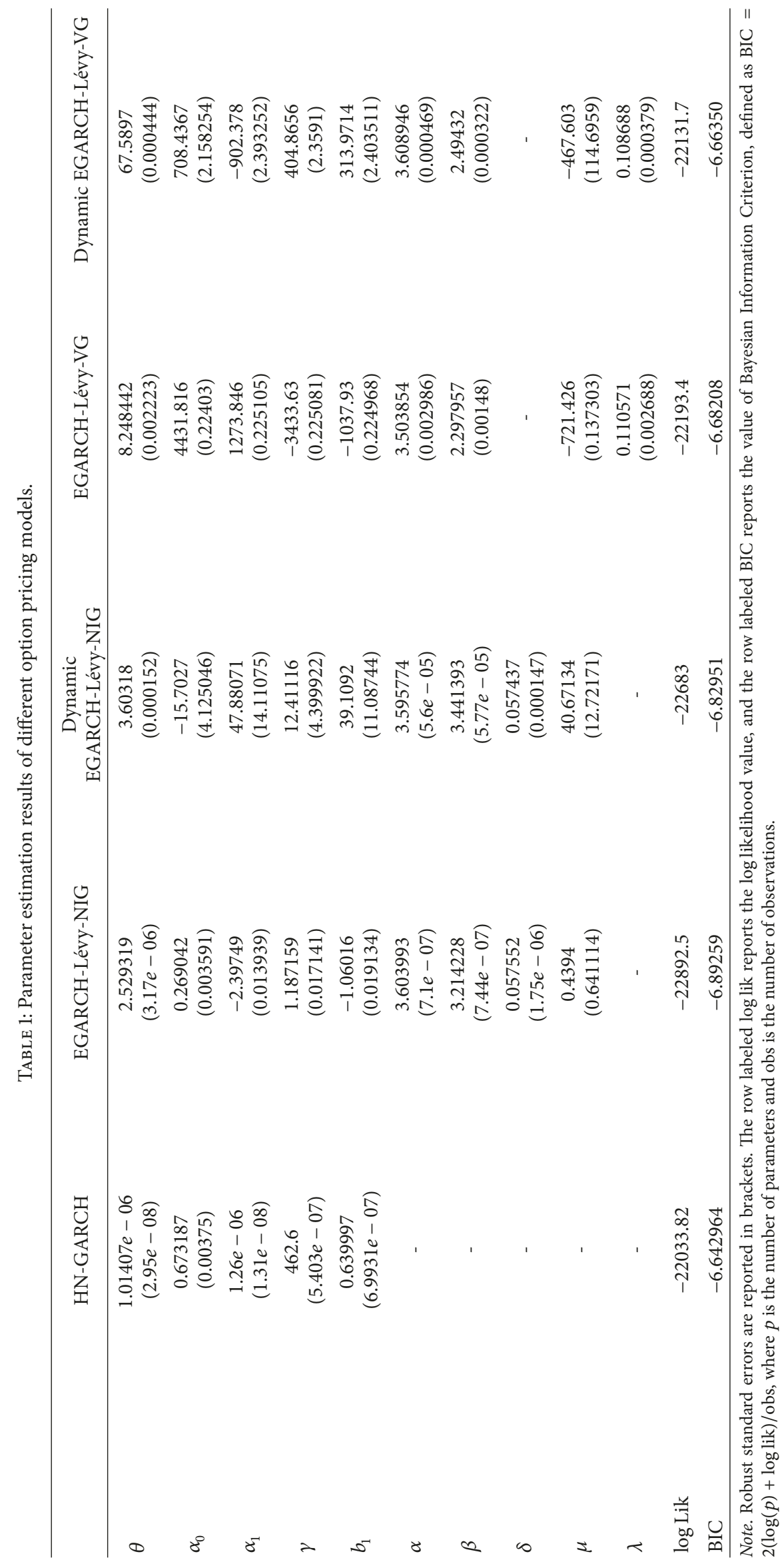


TABLE 2: The error of different option pricing models.

\begin{tabular}{|c|c|c|c|c|}
\hline Model & $T<30$ & $30 \leq T<60$ & $T \geq 60$ & Average \\
\hline HN-GARCH & $40.50 \%$ & $26.52 \%$ & $31.30 \%$ & $32.78 \%$ \\
\hline EGARCH-Lévy-NIG & $51.17 \%$ & $16.41 \%$ & $19.08 \%$ & $28.89 \%$ \\
\hline Dynamic EGARCH- Lévy-NIG & $15.30 \%$ & $13.38 \%$ & $16.58 \%$ & $15.08 \%$ \\
\hline EGARCH-Lévy-VG & $54.70 \%$ & $16.66 \%$ & $19.14 \%$ & $30.17 \%$ \\
\hline Dynamic EGARCH-Lévy-VG & $38.94 \%$ & $13.67 \%$ & $16.59 \%$ & $23.07 \%$ \\
\hline Mean & $40.12 \%$ & $17.33 \%$ & $20.54 \%$ & $25.99 \%$ \\
\hline
\end{tabular}

(II) Generate disturbing term $z_{t}$ submitted to Lévy distribution and generate volatility series $h_{t}$ according to EGARCH-Lévy model. Then generate logarithmic yield series $R_{t+1}^{1}, R_{t+2}^{1}, \ldots, R_{T}^{1}$ under measure $Q$ by using formula (12).

(III) Work out underlying asset's expiry-date price $\left(S_{T}=\right.$ $\left.S_{t} \exp \left(\sum_{i=t+1}^{T} R_{i}^{j}\right)\right)$ under measure $Q$ according to the result specified in section (II) and formula $S_{t+1}=$ $S_{t} \exp \left(R_{t+1}\right)$.

(IV) Repeat steps (II) and (III) for $M(M=10000)$ times and figure out the asset's $M$ expiry-date prices under measure $Q$. Then figure out option worth according to the following formula:

$$
\begin{aligned}
C_{t}= & \exp (-r(T-t)) \frac{1}{M} \\
& \cdot \sum_{j=1}^{M} \max \left(S_{t} \exp \left(\sum_{i=t+1}^{T} R_{i}^{j}\right)-K, 0\right)
\end{aligned}
$$

Theoretical option price can be worked out by following steps (I) to (IV). In order to test option model's pricing error, we use the average relative parameter error (ARPE) index in common use in documents. Its calculating formula is as follows:

$$
\operatorname{ARPE}=\frac{1}{N} \sum_{i=1}^{N} \frac{\left|\widehat{C}_{i}-C_{i}\right|}{C_{i}},
$$

where $\widehat{C}_{i}$ stands for the theoretical price of $i$ th option; $C_{i}$ stands for the market price of $i$ th option; $N$ stands for option number. For more contents of this index, refer to $[10,12]$.

Table 2 shows different models' option pricing errors under the condition of different expiry dates. Its first column specifies model name and the second, third, and fourth columns, respectively, represent different models' pricing errors against short-term options, middle-term options, and long-term options. The fourth column reflects the mean value of different models' pricing errors against options with various time limits. The last line reflects the mean value of five models' pricing errors against short-term options, middleterm options, and long-term options.

According to Table 2, we can draw an important conclusion as follows: for European-style call options, the pricing error of the EGARCH-Lévy model (other than EGARCHLévy-VG, whose pricing error against short-term options is higher) is lower than that of the HN model, indicating that

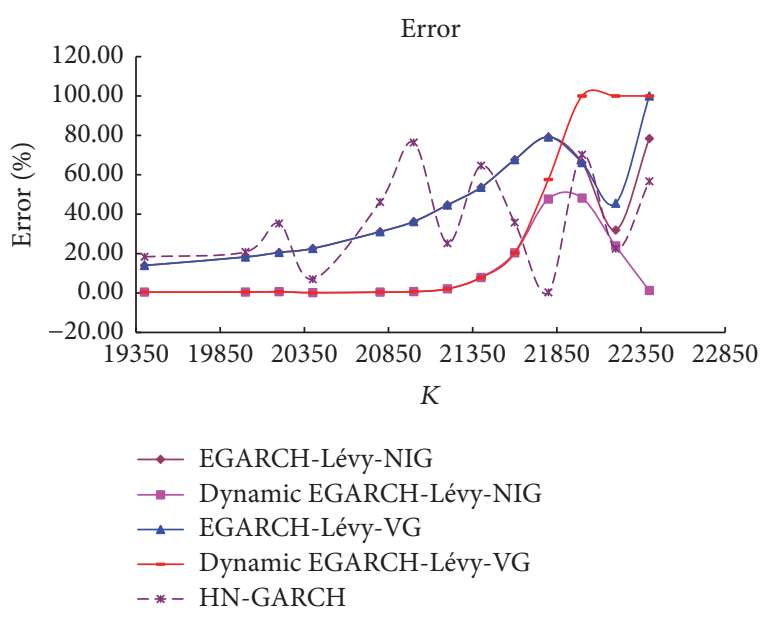

FIGURE 2: The relationship between models' error and exercise price in middle-term options.

the models in this paper can forecast the prices of Europeanstyle options well. With respect to the pricing of short-term options, middle-term options, and long-term options, the pricing error of the Dynamic EGARCH-Lévy-NIG model is the lowest, indicating that this model is better than other models in terms of option pricing. From the last line of Table 2 we can see that all these models' average pricing error against middle-term options is the lowest, the average pricing error against short-term options is the highest, and the average pricing error against long-term options is moderate. This indicates that GARCH option price models can produce a better result when they are used for middle-term options.

Figures 2, 3, and 4 show the relationship between different models' pricing error and exercise price. From Figure 2, we can see that different models' error increases with the increase of exercise price, indicating that the higher the exercise price of option, the weaker the model's capability to estimate option price. The reason for this result is possibly that as exercise price is high, the option is an out-of-the-money option and its price is unstable. When exercise price is low, the error of the EGARCH-Lévy model is much lower than that of the HN model. However, when exercise price is high, the error of the EGARCH-Lévy model is relatively high, indicating that this model should not be used for options whose exercise price deviates seriously from underlying asset's price. For middleterm and long-term options, the EGARCH-Lévy model's pricing error is relatively low. In actual application, such 


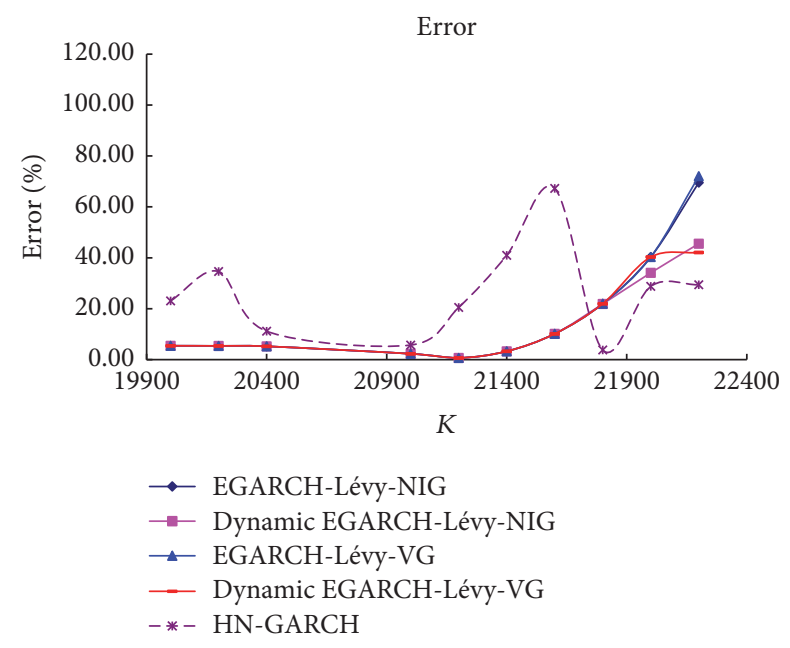

FIGURE 3: The relationship between models' error and exercise price in short-term options.

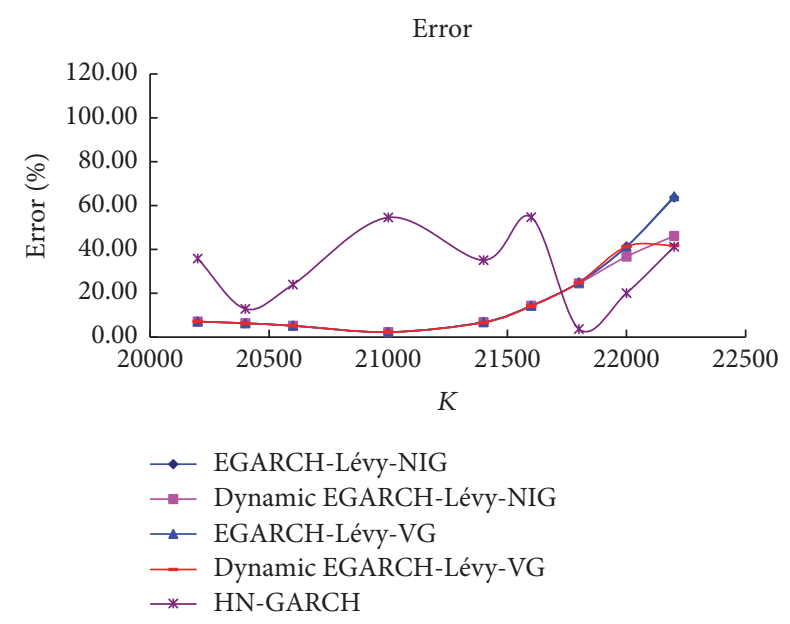

FIGURE 4: The relationship between models' error and exercise price in long-term options.

model may be used for the pricing of middle-term and longterm options.

\section{Main Conclusions}

This paper introduces an option pricing method, which allows asset returns to satisfy a Lévy process. An asymmetrical GARCH model is used to describe assets' random volatility characteristics. Based on the description of dynamic evolution process of asset returns, we use riskneutral martingale pricing technology to build option pricing models and use HSI option data to carry out empirical studies for the EGARCH-Lévy-NIG, EGARCH-Lévy-VG, Dynamic EGARCH-Lévy-NIG, and Dynamic EGARCHLévy-VG models. Results prove that, for short-term, middleterm, and long-term options, the pricing error of the Dynamic EGARCH-Lévy model is the lowest, indicating that this model has an advantage in terms of option pricing. The models introduced in this paper can substantially reduce European-style option pricing errors.

Extended studies in several valuable research directions will be done in the future. One important extension is to introduce option market prices into the estimation of option pricing model parameters to estimate parameters by treating minimum error between option market prices and model prices as objective function so as to increase the accuracy of parameters estimated. Another valuable extension is to introduce the Markov Switching Volatility Model into the description of volatility of asset returns. However, this method needs more complicated estimation technology, for example, Bayesian estimation. Therefore, it is hard to use this method, and our next works would be oriented in some simple and effectiveness methods, such as the state-of-the-art grey system models TPDGM [22], $\operatorname{KGM}(1, n)$ [23], and the hybrid machine learning models [24]. In further studies, Fast Fourier Transform may be used for option pricing so as to increase the efficiency of option pricing models.

\section{Conflicts of Interest}

The authors declare that there are no conflicts of interest.

\section{Authors' Contributions}

The research is designed and performed by Yi Zheng and Gaoxun Zhang. The data was collected by Honglei Zhang. Analysis of data was performed by Gaoxun Zhang and Xinchen Xie. Finally, the paper is written by Yi Zheng, Gaoxun Zhang, Xinchen Xie, and Honglei Zhang. All authors read and approved the final manuscript.

\section{Acknowledgments}

The work was supported in part by the National Natural Science Foundation of China (Grant no. 71702156), the MOE (Ministry of Education in China) Project of Humanities and Social Sciences (Project no. 17YJC630098), the Natural Science Foundation of Southwest University of Science and Technology (no. 15zx7139), the "Chunhui" Plan of Ministry of Education in China (no. S2011012 and no. Z2012017), the Key Scientific Research Fund of Xihua University (Grant no. Z1614417), and the Natural Science Foundation of Education Department of Sichuan (no. 17ZB0414).

\section{References}

[1] F. Black and M. S. Scholes, "The pricing of options corporate liabilities," Journal of Political Economy, vol. 81, pp. 637-659, 1973.

[2] R. C. Merton, “Theory of rational option pricing," Bell Journal of Economics and Management Science, vol. 4, pp. 141-183, 1973.

[3] J. C. Duan, “The garch option pricing model," Mathematical Finance, vol. 5, no. 1, pp. 13-32, 1995.

[4] J. C. Duan, Conditionally fat-tailed distributions and the volatility smile in options, Mimeo, Hong Kong University of Science and Technology, Hong Kong, 1999.

[5] S. L. Heston and S. Nandi, "A closed-form solution for options with stochastic volatility with applications to bond and currency 
options," Review of Financial Studies, vol. 13, no. 3, pp. 585-625, 2000.

[6] L. Stentoft, "Pricing American options when the underlying asset follows GARCH processes," Journal of Empirical Finance, vol. 12, no. 4, pp. 576-611, 2005.

[7] C. Chorro, D. Guegan, and F. Ielpo, A time series approach to option pricing, Springer, Berlin, Germany, 2015.

[8] T. K. Siu, H. Tong, and H. Yang, "On pricing derivatives under GARCH models: a Dynamic Gerber-Shiu approach," North American Actuarial Journal, vol. 8, pp. 17-31, 2004.

[9] P. Christoffersen, C. Dorion, K. Jacobs, and Y. Wang, "Volatility components, affine restrictions, and nonnormal innovations," Journal of Business and Economic Statistics, vol. 28, no. 4, pp. 483-502, 2010.

[10] R. J. Elliott, T. K. Siu, and L. Chan, "Option pricing for GARCH models with Markov switching," International Journal of Theoretical and Applied Finance, vol. 9, no. 6, pp. 825-841, 2006.

[11] P. Christoffersen, K. Jacobs, C. Ornthanalai, and Y. Wang, "Option valuation with long-run and short-run volatility components," Journal of Financial Economics, vol. 90, no. 3, pp. 272297, 2008.

[12] J. V. K. Rombouts and L. Stentoft, "Option pricing with asymmetric heteroskedastic normal mixture models," International Journal of Forecasting, vol. 31, no. 3, pp. 635-650, 2015.

[13] W. K. Newey and D. G. Steigerwald, "Asymptotic bias for quasimaximum-likelihood estimators in conditional heteroskedasticity models," Econometrica, vol. 65, no. 3, pp. 587-599, 1997.

[14] C. Chorro, D. Guegan, F. Ielpo, and H. Lalaharison, “Testing for leverage effect in financial returns," CES Working Papers, 2014.

[15] D. B. Madan and E. Seneta, "The variance Gamma model for share market returns," The Journal of Business, vol. 63, no. 4, pp. 511-524, 1990.

[16] H. Albrecher and M. Predota, "On Asian option pricing for NIG Lévy Processes," Journal of Computational and Applied Mathematics, vol. 172, no. 1, pp. 153-168, 2004.

[17] D. B. Madan and M. Yor, "Representing the CGMY, Meixner Lévy processes as time changed Brownian motions," The Journal of Computational Finance, vol. 12, no. 1, pp. 27-47, 2008.

[18] H. Wu, F. Zhu, and J. Wen, "Option pricing based on conditional infinite pure jump Lévy processes with leverage effect," Journal of Management Sciences in China, vol. 17, no. 8, pp. 74-93, 2014.

[19] O. E. Barndorff-Nielsen and P. Blaesild, "Hyperbolic distributions and ramifications: contributions to theory and application," in Em Statistical Distributions in Scientific Work, vol. 4, pp. 19-44, Reidel, Dordrecht, Netherlands, 1981.

[20] R. F. Engle, "Autoregressive conditional heteroscedasticity with estimates of the variance of United Kingdom inflation," Econometrica, vol. 50, no. 4, pp. 987-1007, 1982.

[21] Z. Ding, C. W. J. Granger, and R. F. Engle, "A long memory property of stock market returns and a new model," Journal of Empirical Finance, vol. 1, no. 1, pp. 83-106, 1993.

[22] X. Ma and Z. Liu, "Application of a novel time-delayed polynomial grey model to predict the natural gas consumption in China," Journal of Computational and Applied Mathematics, vol. 324, pp. 17-24, 2017.

[23] X. Ma and Z. Liu, "The kernel-based nonlinear multivariate grey model," Applied Mathematical Modelling, vol. 56, pp. 217-238, 2018.

[24] X. Ma and Z. Liu, "Predicting the oil production using the novel multivariate nonlinear model based on Arps decline model and kernel method," Neural Computing and Applications, pp. 1-13, 2016. 


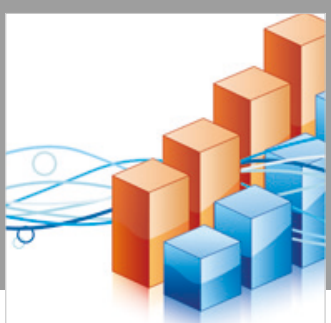

Advances in

Operations Research

\section{-n-m}
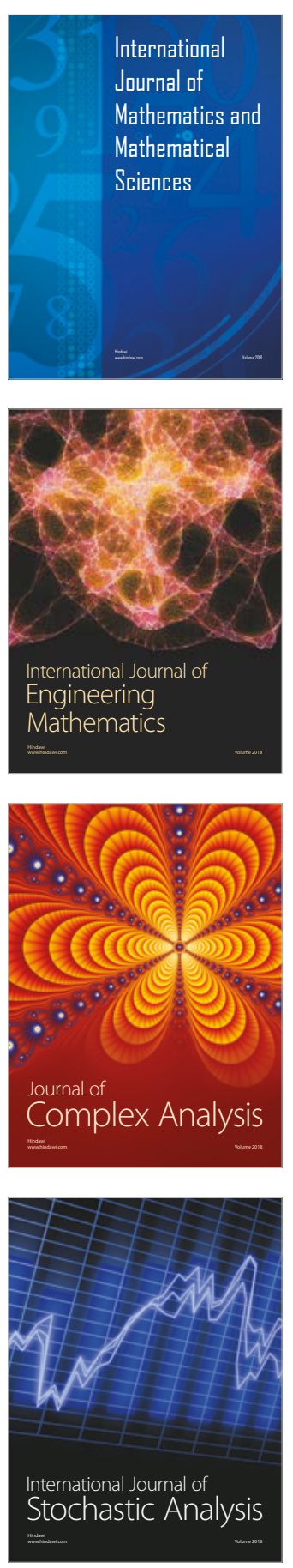
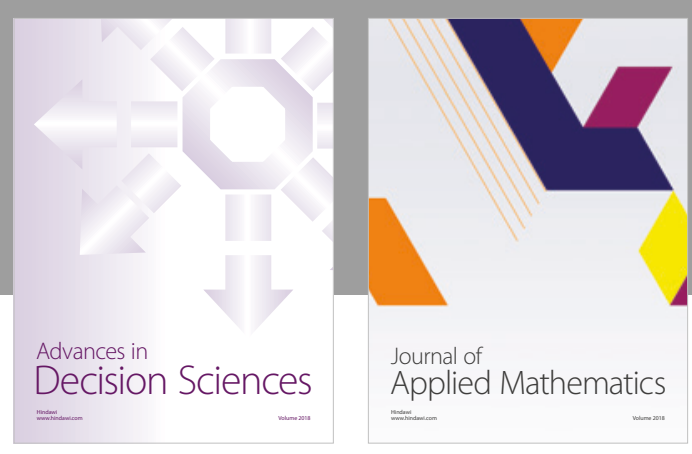

Journal of

Applied Mathematics
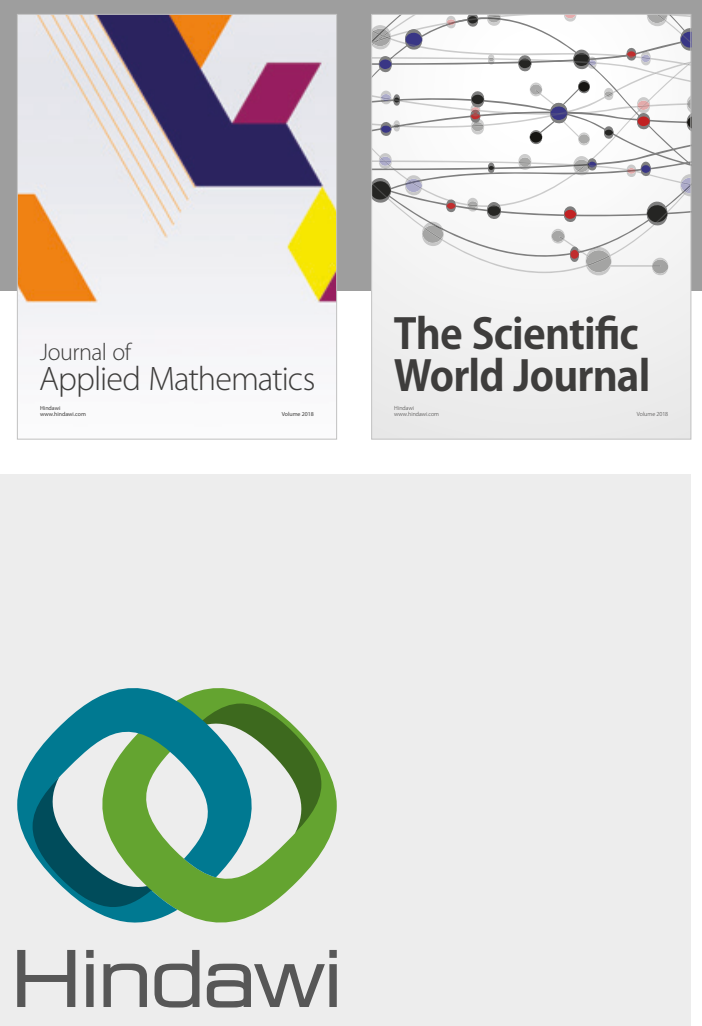

Submit your manuscripts at

www.hindawi.com

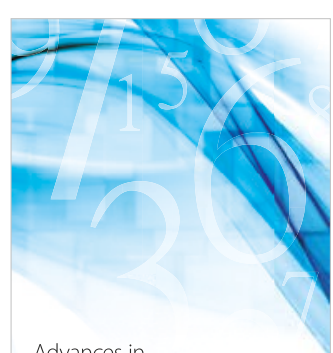

Advances in
Numerical Analysis
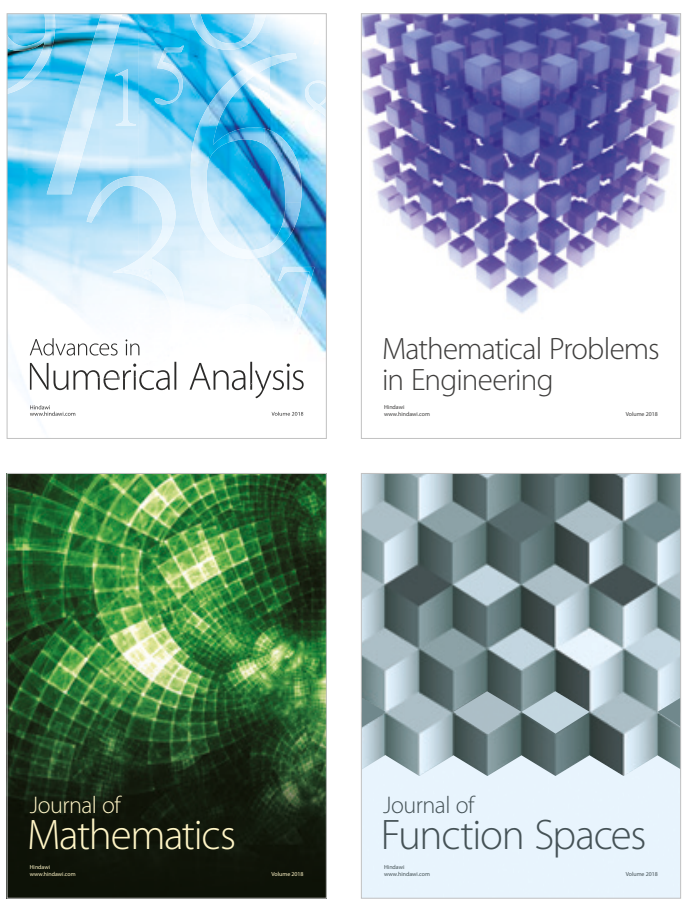

Mathematical Problems in Engineering

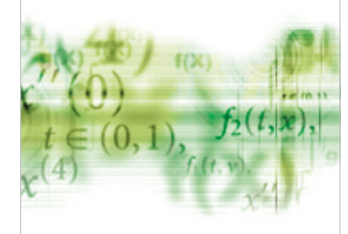

International Journal of

Differential Equations

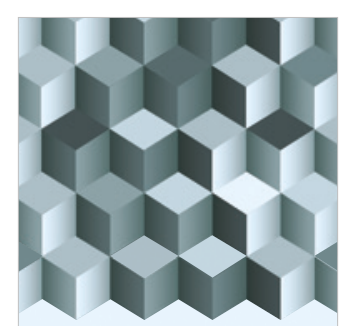

Journal of

Function Spaces

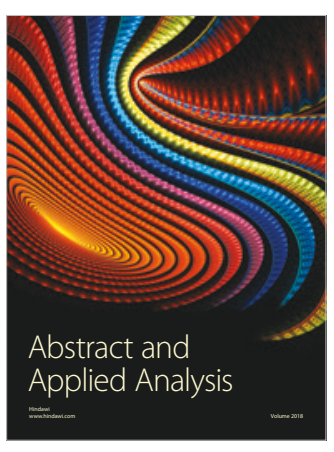

The Scientific

World Journal

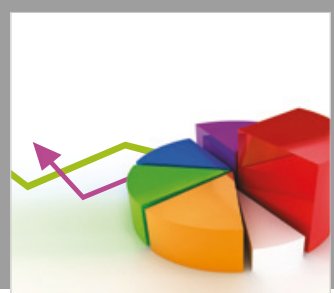

Journal of

Probability and Statistics
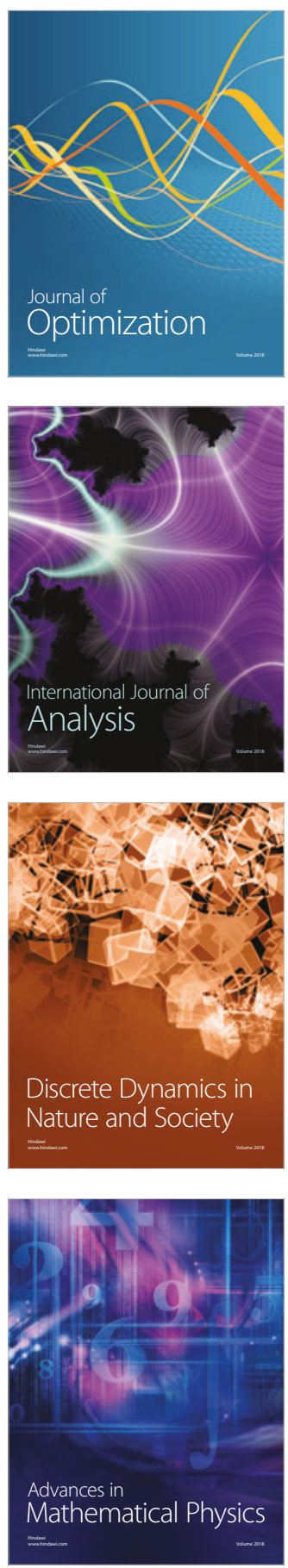